\title{
A Pairing Strategy for Tic-Tac-Toe on the Integer Lattice with Numerous Directions
}

\author{
Klay Kruczek \\ Mathematics Department \\ Western Oregon University \\ Monmouth, OR 97361 \\ kruczekk@wou.edu
}

\author{
Eric Sundberg \\ Mathematics Department \\ Occidental College \\ Los Angeles, CA 90041 \\ sundberg@oxy .edu
}

Submitted: Jan 16, 2008; Accepted: Nov 10, 2008; Published: Nov 24, 2008

Mathematics Subject Classification: 91A46

\begin{abstract}
We consider a tic-tac-toe game played on the $d$-dimensional integer lattice. The game that we investigate is a Maker-Breaker version of tic-tac-toe. In a MakerBreaker game, the first player, Maker, only tries to occupy a winning line and the second player, Breaker, only tries to stop Maker from occupying a winning line. We consider the bounded number of directions game, in which we designate a finite set of direction-vectors $\mathcal{S} \subset \mathbb{Z}^{d}$ which determine the set of winning lines. We show by a simple pairing strategy that Breaker can win this game if the length of each winning line is at least $3|\mathcal{S}|$. It should be noted that Breaker's winning strategy can be used as a drawing strategy for Player 2 in the strong version of this game.
\end{abstract}

\section{Introduction}

The traditional game of $3 \times 3$ tic-tac-toe is a type of positional game. In particular, $3 \times 3$ tic-tac-toe is an example of what we call a strong positional game. In general, a positional game [1] is a two-person game with complete information played on a hypergraph $(V, \mathcal{H})$, where $V$ is an arbitrary set, called the board of the game, and $\mathcal{H}$ is a family of subsets of $V$, called the winning sets. The two players, Player 1 and Player 2, alternately occupy previously unoccupied elements of $V$. In a strong positional game, the first player to occupy all points of some winning set wins. We say that Player 1 has a winning strategy if no matter what Player 2 does, Player 1 can follow that strategy to win the game. If neither player has a winning strategy, we say that the game is a draw.

The traditional game of $3 \times 3$ tic-tac-toe is an example of a strong positional game where the nine positions are the vertices and the eight lines (3 vertical, 3 horizontal, and 2 diagonal) are the winning sets. Most people are aware that $3 \times 3$ tic-tac-toe is a draw game. 
By using a strategy stealing argument [1], it can be shown that in all strong positional games, either Player 1 has a winning strategy or the game is a draw. Thus, it is reasonable to consider an alternate positional game in which it is possible for Player 2 to have a winning-strategy. One such game is the Maker-Breaker game. A Maker-Breaker positional game is where the first player, Maker, only tries to occupy winning sets, and the second player, Breaker, only tries to stop Maker from doing so. Thus, in a Maker-Breaker positional game, Maker wins if she occupies all points of some winning set, and Breaker wins if he prevents Maker from doing so. Therefore, by definition, someone always wins in a Maker-Breaker positional game (there are no draws). It is interesting to note that when $3 \times 3$ tic-tac-toe is played as a Maker-Breaker positional game, Maker has a winning strategy, as Maker does not need to block Breaker from obtaining a winning line.

Since we will be considering a semi-infinite game, i.e., a game where $|V|=\infty,|\mathcal{H}|=$ $\infty$, yet $\forall A \in \mathcal{H},|A|<\infty$, we should describe what constitutes a win for Breaker in such a game. If a Maker-Breaker game is played on a semi-infinite hypergraph $(V, \mathcal{H})$, then we say that Breaker has a winning strategy on $(V, \mathcal{H})$ if for all $j \in \mathbb{Z}^{+}$, Breaker can prevent Maker from completely occupying a winning set by turn $j$.

We will consider the following Maker-Breaker game on $\mathbb{Z}^{d}$. The vertices of the board are all the points of $\mathbb{Z}^{d}$. Each winning line has length $m$, and the directions of the winning lines are determined by a set of vectors $\mathcal{S}$, which we will call the set of direction vectors. We will require that for each $\vec{v} \in \mathcal{S}$, the greatest common divisor of its coordinates is 1 , which we will denote by $\operatorname{gcd}(\vec{v})=1$. Also, for each vector $\vec{v} \in \mathcal{S}$, the vector $-\vec{v} \notin \mathcal{S}$, since $\vec{v}$ and $-\vec{v}$ will determine the same set of winning lines. This way we can say that the set of winning lines is

$$
\left\{\{\vec{p}, \vec{p}+\vec{v}, \vec{p}+2 \vec{v}, \ldots \vec{p}+(m-1) \vec{v}\}: \vec{p} \in \mathbb{Z}^{d}, \vec{v} \in \mathcal{S}\right\} .
$$

We refer to this game as $M B_{\mathcal{S}}^{d}(m)$.

Our main result will be to show that if the length $m$ of each winning line is at least $3|\mathcal{S}|$, then Breaker can win $M B_{\mathcal{S}}^{d}(m)$ by using a simple pairing strategy. Our strategy is essentially a generalization of a Player 2's pairing strategy given by Beck [1] (Chapter 10) for the 12-in-a-row game played on $\mathbb{Z} \times \mathbb{Z}$. The Maker-Breaker version of this game is $M B_{\mathcal{S}}^{2}(12)$, where $\mathcal{S}=\{(0,1),(1,0),(1,1),(1,-1)\}$. In the pairing strategy employed by Beck in the strong game, Player 2 assigns a direction to each point by "tiling" $\mathbb{Z} \times \mathbb{Z}$ with $4 \times 4$ blocks which contain the direction assignment shown in Figure 1 . Then Player 2 pairs off the points of $\mathbb{Z} \times \mathbb{Z}$ so that each point is paired with a unique sibling from a different block. Two siblings have the properties that their coordinates are congruent modulo 4 (thus they are assigned the same direction), they lie on a line whose direction matches their assigned direction, and they are as close to each other as possible (so they are only 4 "steps" away from each other). Whenever Player 1 occupies a point, Player 2 immediately responds by occupying the sibling of that point. Thus, Player 1 can occupy at most 11 points in a row. Because a drawing strategy for Player 2 in a strong game is the same as a winning strategy for the Maker-Breaker version of the same game, Breaker has a winning strategy in $M B_{\mathcal{S}}^{2}(12)$.

Our result extends this idea by revealing that this strategy can be generalized to the 


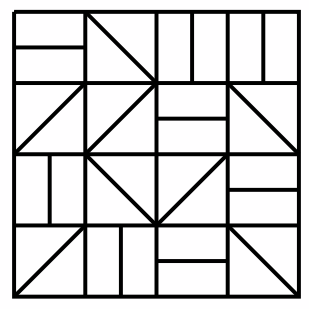

Figure 1: An assignment of directions

$M B_{\mathcal{S}}^{d}(m)$ game, where $\mathcal{S}$ is an arbitrary finite set of direction vectors, and interestingly, it shows that the length of the winning lines that Breaker is able to block only depends of $|\mathcal{S}|$, not on the particular vectors that are in $\mathcal{S}$. Thus, our generalization shows that Breaker also can use essentially the same pairing strategy to win the $M B_{\mathcal{S}^{\prime}}^{2}(12)$ game, where $\mathcal{S}^{\prime}=\left\{(1,0),\left(1,2^{100}\right),\left(2^{100}, 3^{100}\right),\left(5^{100 !}, 1\right)\right\}$, as he used to win the Maker-Breaker 12-in-a-row game.

\section{Main Theorem}

Theorem 1 In the Maker-Breaker game on $\mathbb{Z}^{d}$ where there is a finite set $\mathcal{S} \subset \mathbb{Z}^{d}$ of winning line direction-vectors, Breaker has a pairing strategy that allows him to win if the length of each winning line is at least $3|\mathcal{S}|$, i.e., Breaker has a winning pairing-strategy for $M B_{\mathcal{S}}^{d}(m)$ if $m \geq 3|\mathcal{S}|$.

Proof: Let $n=|\mathcal{S}|$. We partition $\mathbb{Z}^{d}$ into disjoint sub-boards comprised of hypercubes of size $n^{d}$ so that one of the sub-boards, which we call $B$, has $(0,0, \ldots, 0)$ and $(n-1, n-$ $1, \ldots, n-1)$ as its diagonal corners, and if we work $\bmod n$, then every other sub-board is equivalent to $B$. We can then view the points of an individual sub-board as the elements of the additive group $\mathbb{Z}_{n}^{d}$. Using this perspective, we can see that when working mod $n$, each direction-vector $\vec{v} \in \mathcal{S}$ determines a line of length $n$ through the points $\overrightarrow{0}$ and $\vec{v}$. This line has length $n$ because $\operatorname{gcd}(\vec{v})=1$ and therefore the subgroup generated by $\vec{v}$, namely $\{\overrightarrow{0}, \vec{v}, 2 \vec{v}, 3 \vec{v}, \ldots\}$, has order $n$. For each $\vec{v} \in \mathcal{S}$ there are $n^{d-1}$ cosets associated with the subgroup generated by $\vec{v}$, where each coset corresponds to a distinct line of length $n$ with direction $\vec{v}$ that is contained in the $n^{d}$ sub-board when working $\bmod n$. Since there are $n$ different direction-vectors, there is a total of $n \cdot n^{d-1}$ distinct lines that pass through the $n^{d}$ sub-board when working $\bmod n$. Clearly, there are $n^{d}$ points in the sub-board $B$, so Breaker wants to assign a direction to each point in $B$ so that for each line there is a unique point contained in that line whose assigned direction matches the direction of the line. More formally, let $\mathrm{L}$ be the set of lines and $\mathcal{P}$ the set of points in $B$. Define the function $D_{\mathrm{L}}: \mathrm{L} \rightarrow \mathcal{S}$ so that for each line $l, D_{\mathrm{L}}(l)$ is the direction of that line. We will then construct a function $D_{\mathcal{P}}: \mathcal{P} \rightarrow \mathcal{S}$ so that for each line $l$ in $B$, there exists a unique point $p \in l$ such that $D_{\mathcal{P}}(p)=D_{\mathrm{L}}(l)$. Thus we are faced with a matching problem. 
To solve this matching problem, we can create a bipartite graph $G=(P, E, L)$ where $P$ corresponds to the set of points $\mathcal{P}$ and $L$ corresponds to the set of lines $\mathrm{L}$, and a point $p \in P$ is adjacent to a line $l \in L$ if and only if point $p$ is contained in line $l$ when we view them as elements of the $n^{d}$ sub-board. Every point $p$ will have degree $n$, since, for each direction-vector $\vec{v}$, there is a line that contains $p$ and has direction-vector $\vec{v}$. Likewise, every line $l$ will have degree $n$, since each line contains exactly $n$ points. Therefore we have an $n$-regular bipartite graph, and by a corollary to Hall's Marriage theorem, there exists a perfect matching in $G$. We can fix a particular perfect matching $M$, then assign directions to the points so that $D_{\mathcal{P}}(p):=D_{\mathrm{L}}(l)$ if and only if $p$ is adjacent to $l$ in the matching $M$. Once this direction assignment is established for the points of $B$, we repeat this assignment for every other equivalent $n^{d}$ sub-board in the partition of $\mathbb{Z}^{d}$, i.e., we extend the function $D_{\mathcal{P}}$ so that $D_{\mathcal{P}}: \mathbb{Z}^{d} \rightarrow \mathcal{S}$ using the following procedure. Let $\vec{q}$ be a point in $\mathbb{Z}^{d} \backslash B$. We can uniquely write $\vec{q}=n \vec{p}+\vec{t}$, where $\vec{t} \in B$, and we define $D_{\mathcal{P}}(\vec{q}):=D_{\mathcal{P}}(\vec{t})$. Likewise we redefine $\mathrm{L}$ to be the set of all lines in $\mathbb{Z}^{d}$ with directions from $\mathcal{S}$ and define $D_{\mathrm{L}}: \mathrm{L} \rightarrow \mathcal{S}$ in the obvious way.

We then pair points together so that two points $\vec{p}_{1}$ and $\vec{p}_{2}$ are paired together only if:

- $\vec{p}_{1} \equiv \vec{p}_{2} \quad(\bmod n)$

- there exists $l \in \mathrm{L}$ such that $\vec{p}_{1}, \vec{p}_{2} \in l$ and $D_{\mathrm{L}}(l)=D_{\mathcal{P}}\left(\vec{p}_{1}\right)=D_{\mathcal{P}}\left(\vec{p}_{2}\right)$;

- and they are "as close to each other as possible."

Let us now describe one systematic way to pair together points in $\mathbb{Z}^{d}$. Let $\vec{q}$ be a point in $\mathbb{Z}^{d}$. Recall that we can uniquely write $\vec{q}=n \vec{p}+\vec{t}$, where $\vec{t} \in B$ and $\vec{p}=\left(p_{1}, \ldots, p_{d}\right)$. Let $\vec{v}=\left(v_{1}, \ldots, v_{d}\right)$ be the direction-vector assigned to $\vec{q}$, i.e., $D_{\mathcal{P}}(\vec{q})=\vec{v}$. Let $v_{i}$ be the first nonzero coordinate of $\vec{v}$. We may assume $v_{i}>0$ since the direction-vector $\vec{v}$ determines the same set of lines as the direction-vector $-\vec{v}$. Now suppose that $p_{i} \equiv c \quad\left(\bmod 2 v_{i}\right)$, where $0 \leq c<2 v_{i}$. We say $\vec{q}$ is low if $0 \leq c<v_{i}$, and $\vec{q}$ is high if $v_{i} \leq c<2 v_{i}$. Then $\vec{q}$ gets paired with $\vec{q}+n \vec{v}$ if and only if $\vec{q}$ is low. It should be clear that $\vec{q}$ is low if and only if $\vec{q}+n \vec{v}$ is high. Thus, if $\vec{q}$ is high, then $\vec{q}$ is paired with $\vec{q}-n \vec{v}$.

We now have to show that this pairing allows Breaker to stop Maker from completely occupying any line of length $3 n$ and longer. Let $\ell$ be a line of length $m \geq 3 n$, with direction-vector $\vec{v}$, and starting point $\vec{q}$. We may write $\ell=\{\vec{q}+j \vec{v}: 0 \leq j \leq m-1\}$. Because of how Breaker assigned direction-vectors to the points of $\mathbb{Z}^{d}$, each line $l$ of length $n$ contains exactly one point $p$ such that $D_{\mathcal{P}}(p)=D_{\mathrm{L}}(l)$. Thus, for the line $\ell$, there is a unique integer $j$ such that $0 \leq j \leq n-1$ and $D_{\mathcal{P}}(\vec{q}+j \vec{v})=D_{\mathrm{L}}(\ell)=\vec{v}$. Because Breaker assigns the same direction-vector to points that are equivalent mod $n$, $D_{\mathcal{P}}(\vec{q}+(n+j) \vec{v})=\vec{v}$ and $D_{\mathcal{P}}(\vec{q}+(2 n+j) \vec{v})=\vec{v}$. Notice that because $0 \leq j \leq n-1$, we have $0 \leq n i+j \leq 3 n-1$ for $i=0,1,2$. Thus, since $m \geq 3 n$, we know that $\vec{q}+j \vec{v}, \vec{q}+(n+j) \vec{v}$, and $\vec{q}+(2 n+j) \vec{v}$ are all contained in line $\ell$. If $\vec{q}+(n+j) \vec{v}$ is low, then Breaker will have paired it with $\vec{q}+(2 n+j) \vec{v}$, otherwise it is high and he will have paired it with $\vec{q}+j \vec{v}$. In either case, Breaker will eventually occupy one of those three points from $\ell$ and therefore block line $\ell$. Since $\ell$ was an arbitrary line with at least $3 n$ points on it, we see that Maker can never occupy a line of length $m \geq 3 n$. This concludes the proof of Theorem 1 . 


\section{Conclusion}

We have shown that in the $M B_{\mathcal{S}}^{d}(m)$ game where $m \geq 3|\mathcal{S}|$, Breaker has a winning pairing strategy. This is by no means a tight bound. There are certain versions of the $M B_{\mathcal{S}}^{d}$ game where it is known that Breaker can do better than blocking all lines of length at least $3|\mathcal{S}|$. For example, Guy, Selfridge, and Zetters [4] show that Breaker can block all lines of length 8 and longer in Maker-Breaker tic-tac-toe on $\mathbb{Z} \times \mathbb{Z}$ with the standard four directions $\{(1,0),(0,1),(1,1),(1,-1)\}$. Another example which illustrates the slack in the bound can be constructed by restricting, in a natural way, the types of direction-vectors that are contained in $\mathcal{S}$.

Consider the classic $3 \times 3$ tic-tac-toe game and the $n^{d}$ tic-tac-toe games. Each directionvector $\vec{v}$ in those games has the property that the magnitude of each of its coordinates is at most 1, i.e., if $\vec{v}=\left(v_{1}, \ldots, v_{d}\right)$ is a direction-vector, then $\left|v_{i}\right| \leq 1$ for $1 \leq i \leq d$. A logical generalization of this game is to consider a tic-tac-toe game played on $\mathbb{Z}^{d}$ where the set of direction-vectors $\mathcal{S}$ contains all direction-vectors (i.e., vectors $\vec{v}$ with $\operatorname{gcd}(\vec{v})=1$ ) such that their coordinates satisfy $\left|v_{i}\right| \leq k$ for $1 \leq i \leq d$. In this game, by using a strategy based on the Erdős-Selfridge theorem [3], we are able to show that Breaker can block lines whose length is on the order of $d^{2} \log (k d)$. These results will appear in a future paper. If Breaker were to use the pairing strategy from Theorem 1 instead, he could only block lines whose length is on the order of $(2 k)^{d}$. (This is because there will be on the order of $(2 k)^{d}$ different direction-vectors in $\mathcal{S}$.) Clearly, in this instance, the Erdős-Selfridge-based strategy is much better.

However, there is still a lot of value in the pairing strategy result from Theorem 1 since it is more suitable for other problems. For example, consider Maker-Breaker tic-tac-toe on $\mathbb{Z} \times \mathbb{Z}$ with $\mathcal{S}=\left\{(1,0),\left(1,2^{j}\right)\right\}$ where $j \in \mathbb{Z}$ is some large constant. Since there are only two direction-vectors, the pairing strategy from Theorem 1 allows Breaker to block all winning lines of length 6 and longer. However, if we tried to directly apply the ErdösSelfridge-based strategy, since the coordinates of the direction-vectors are bounded by $2^{j}$, Breaker would only be able to block lines whose size is on the order of $j$.

Also, the pairing strategy from Theorem 1 is extremely simple to describe and could be easily used in practice. All it requires Breaker to do is pair off points in the beginning, and then at each turn, his next move is immediately determined after Maker moves. In the Erdős-Selfridge-based strategy, at each turn Breaker has to calculate which point would remove the most potential from the board, where potential refers to a measurement of how dangerous it would be for Maker to occupy that point. We conclude by mentioning a conjecture.

Conjecture 1 If $m \geq 2|\mathcal{S}|+1$, then Breaker has a winning pairing-strategy for $M B_{\mathcal{S}}^{d}(m)$.

Our conjecture is based on a pairing-strategy given by Hales and Jewett [1], [2] for the tic-tac-toe game played on $\mathbb{Z} \times \mathbb{Z}$ with the four standard directions. They tile the integer lattice with the $8 \times 8$ direction assignment shown in Figure 2 . The direction assignment is constructed so that the two points assigned to a line are adjacent points from that line. With this assignment, Breaker can block all lines of length 9 and longer in Maker-Breaker 
tic-tac-toe on $\mathbb{Z} \times \mathbb{Z}$ with the standard directions $\{(1,0),(0,1),(1,1),(1,-1)\}$. We believe that this idea of adjacent point pairing can be generalized to the $M B_{\mathcal{S}}^{d}(m)$ game.

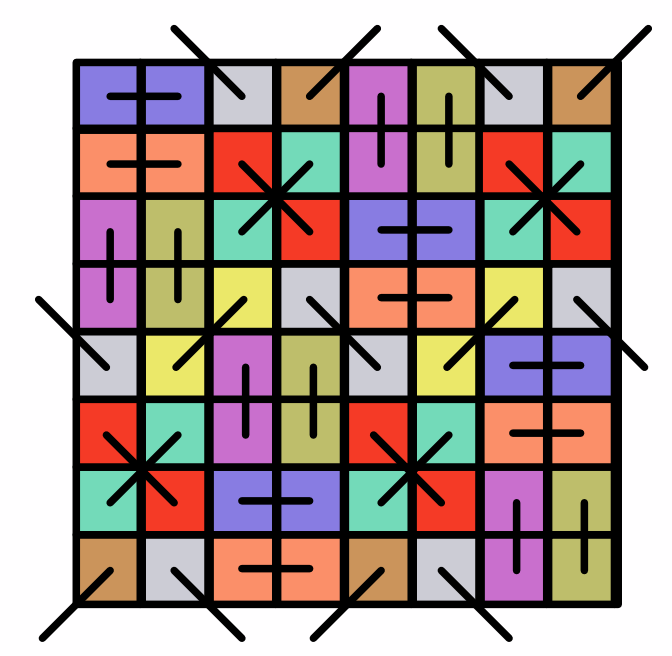

Figure 2: The Hales and Jewett $8 \times 8$ direction assignment.

\section{References}

[1] József Beck. Combinatorial Games: Tic-Tac-Toe Theory. Cambridge University Press, 2008.

[2] Elwyn R. Berlekamp, John H. Conway, and Richard K. Guy. Winning ways for your mathematical plays. Vol. 2. Academic Press Inc. [Harcourt Brace Jovanovich Publishers], London, 1982. Games in particular.

[3] P. Erdős and J. L. Selfridge. On a combinatorial game. J. Combinatorial Theory Ser. A, 14:298-301, 1973.

[4] Richard K. Guy; J. L. Selfridge; T. G. L. Zetters. Solutions of problems dedicated to Emory P. Starke. The American Mathematical Monthly, 87(7):575-576, 1980. 